\title{
Brane cosmological solutions in six-dimensional warped flux compactifications
}

\author{
Tsutomu Kobayashi1,* and Masato Minamitsuji 2 , 田 \\ ${ }^{1}$ Department of Physics, Waseda University, Okubo 3-4-1, Shinjuku, Tokyo 169-8555, Japan \\ ${ }^{2}$ Arnold-Sommerfeld-Center for Theoretical Physics, Department für Physik, \\ Ludwig-Maximilians-Universität, Theresienstr. 37, D-80333, Munich, Germany
}

\begin{abstract}
We study cosmology on a conical brane in the six-dimensional Einstein-Maxwell-dilaton system, where the extra dimensions are compactified by a magnetic flux. We systematically construct exact cosmological solutions using the fact that the system is equivalently described by $(6+n)$ dimensional pure Einstein-Maxwell theory via dimensional reduction. In particular, we find a powerlaw inflationary solution for a general dilatonic coupling. When the dilatonic coupling is given by that of Nishino-Sezgin chiral supergravity, this reduces to the known solution which is not inflating. The power-law solution is shown to be the late-time attractor. We also investigate cosmological tensor perturbations in this model using the $(6+n)$-dimensional description. We obtain the separable equation of motion and find that there always exist a zero mode, while tachyonic modes are absent in the spectrum. The mass spectrum of Kaluza-Klein modes is obtained numerically.
\end{abstract}

PACS numbers: 04.50.+h, 98.80.Cq

\section{INTRODUCTION}

Higher dimensional models have a longstanding history, but there has in particular been a growing interest since the discovery of D-branes in string theory, leading to the concept of "braneworld" scenarios. The braneworld framework allows us to consider large (or even infinite) extra dimensions due to localization of matter fields on the brane [1]. The brane models with two extra-dimensions have drawn considerable attention among others. This is because sixdimensional (6D) models may be able to eliminate the hierarchy problem in particle phenomenology, and at the same time bring us the possibility to detect signatures of extra dimensions, as the compactification radius can be of order $0.1 \mathrm{~mm}[2]$. Moreover, recent realizations of such brane models with rugby-ball (or football) shaped extra-dimensions provide a possible self-tuning mechanism to resolve the cosmological constant problem [3], though the mechanism has been criticized for several reasons [4].

In contrast to the 5D (codimension 1) brane models (e.g., [ [5, $[6]$ ), cosmological aspects of $6 \mathrm{D}$ models have not been explored much (e.g., 7, 8] ). Taking into account the gravitational backreaction of branes, one immediately faces the problem of the localization of matter: it is difficult for a codimension 2 defect to accommodate energy-momentum tensor different from pure tension. This fact hampers attempts to construct 6D models for which 3-branes have Friedmann-Robertson-Walker geometry. One will notice, however, that not only brane localized matter fields but also those in the bulk can support a cosmic expansion of branes. We focus here on this latter possibility. ${ }^{1}$

The purpose of this paper is to present exact cosmological solutions in the presence of a scalar field, flux, and conical 3 -branes in six dimensions. We will be considering the Einstein-Maxwell-dilaton system, and for a certain choice of a parameter our Lagrangian reduces to that of Nishino-Sezgin chiral supergravity [17, 18]. In Ref. [19], Mukohyama et al. presented a warped braneworld configuration in a more simplified setup in Einstein-Maxwell theory. The perturbation dynamics and stability issue have been extensively studied in [20, 21, 22]. In the context of NishinoSezgin supergravity, similar warped compactification solutions with Minkowski branes were found in [23, 24, 25] and de Sitter braneworlds were explored in [26]. While none of these solutions have a nontirivial time-dependence of metric functions, Tolley et al. 27] found cosmological scaling solutions in 6D chiral supergravity without showing explicitly the metric of the internal space (see also Ref. [28] for a numerical construction of cosmological solutions in Nishino-Sezgin supergravity). The solutions given in this paper are exact both for the noncompact four dimensions and for the compact internal space. After constructing our cosmological solutions, we shall also discuss the behavior of tensor perturbations in the present model.

We will be taking the approach developed in Ref. [29], which was originally used to study 5D warped brane

\footnotetext{
*Email: tsutomu" at" gravity.phys.waseda.ac.jp

†Email: masato" at"theorie.physik.uni-muenchen.de

1 One of the possible ways out is to regularize conical deficits [9, 10, 11, 12, 13, 14, 15] so as to put ordinary matter on the brane. Changing the bulk gravity theory (e.g., [16]) also helps.
} 
models with a scalar field [30, 31, 32, 33, 34, 35]. In [29], instead of solving the relevant 5D field equations, the authors employed a $(5+n)$-dimensional equivalent description without a scalar field. This approach, with the extra $n$-dimensional space playing effectively the role of the scalar field after dimensional reduction, greatly simplifies the analysis of cosmological perturbations as well as constructing dynamical background solutions. Here we use a $(6+n)-$ dimensional Einstein-Maxwell system instead of working directly in the 6D model with a scalar field, and show that the same mathematical technique as [29] successfully works.

The structure of the paper is as follows. In the next section we introduce our dimensional reduction approach and demonstrate how one can obtain a Minkowski braneworld using the $(6+n)$-dimensional description. In Sec. III we construct cosmological solutions in the $6 \mathrm{D}$ braneworld. The behavior of tensor perturbations is discussed in Sec. IV. Finally, our conclusions are summarized in Sec. V.

\section{DIMENSIONAL REDUCTION APPROACH TO 6D BRANEWORLD MODELS}

We are interested in the $6 \mathrm{D}$ system described by the action

$$
S^{(6)}=\int d^{6} x \sqrt{-g}\left[\frac{M_{(6)}^{4}}{2}\left(R[g]-\partial_{a} \varphi \partial^{a} \varphi-2 e^{-\gamma \varphi} \Lambda\right)-\frac{1}{4} e^{\gamma \varphi} F_{a b} F^{a b}\right],
$$

with two or fewer branes. Here $F_{a b}$ denotes the $U(1)$ field strength. For $\gamma=1$ the action (2.1) coincides with the bosonic part of Nishino-Sezgin supergravity [17, 18] with some fields set to be zero consistently (and with the identification of $\Lambda \rightarrow 2 g_{1}^{2} M_{(6)}^{4}$, where $g_{1}$ is the $U(1)$ gauge coupling). In this paper we consider more general cases and assume that the parameter $\gamma$ is arbitrary in the range $0 \leq \gamma \leq 1$.

The above Einstein-Maxwell-dilaton system with branes has an equivalent $(6+n)$-dimensional description in terms of pure Einstein-Maxwell theory. To show this, we start with the $(6+n)$-dimensional Einstein-Maxwell action

$$
S^{(6+n)}=\int d^{6+n} \mathcal{X} \sqrt{-\mathcal{G}}\left[\frac{M_{(6+n)}^{4+n}}{2}(\mathcal{R}[\mathcal{G}]-2 \Lambda)-\frac{1}{4} \mathcal{F}_{M N} \mathcal{F}^{M N}\right],
$$

and the $(4+n)$-dimensional pure tension branes $(i=1,2)$

$$
S_{\mathrm{b} i}^{(4+n)}=-\int d^{4+n} \mathcal{X} \sqrt{-\mathcal{G}_{\mathrm{b} i}} \mathcal{T}_{i}
$$

where $\mathcal{G}_{\mathrm{b} i}$ is the determinant of the induced metric on the brane.

Let us consider the metric of the form

$$
\mathcal{G}_{M N} d \mathcal{X}^{M} d \mathcal{X}^{N}=\underbrace{e^{-n \phi(x) / 2} g_{a b}(x) d x^{a} d x^{b}}_{6 \mathrm{D}}+\underbrace{e^{2 \phi(x)} \delta_{m n} d y^{m} d y^{n}}_{n \mathrm{D}},
$$

and the field strength with

$$
\mathcal{F}_{a b}=\mathcal{F}_{a b}(x) \text { and } \mathcal{F}_{m M}=0 .
$$

With the above ansatz, dimensional reduction yields

$$
S^{(6)}=\int d^{6} x \sqrt{-g}\left[\frac{M_{(6)}^{4}}{2}\left(R[g]-\frac{n(n+4)}{4} \partial_{a} \phi \partial^{a} \phi-2 e^{-n \phi / 2} \Lambda\right)-\frac{1}{4} e^{n \phi / 2} F_{a b} F^{a b}\right],
$$

where $M_{(6)}^{4}:=M_{(6+n)}^{4+n} \mathcal{V}_{(n)}, F_{a b}:=\mathcal{F}_{a b} \mathcal{V}_{(n)}^{1 / 2}$, and $\mathcal{V}_{(n)}$ is the volume of the $n$-dimensional flat space. Note that in (2.6) indices are raised and lowered with $g_{a b}$. Now, defining

$$
\varphi:=\frac{\sqrt{n(n+4)}}{2} \phi, \quad \gamma:=\sqrt{\frac{n}{4+n}},
$$

we see that (2.6) is identical to the action (2.1). We also define the 3 -brane tension as $T_{i}:=\mathcal{T}_{i} \mathcal{V}_{(n)}$. Then the brane action (2.3) reduces to

$$
S_{\mathrm{b} i}^{(4)}=-\int d^{4} x \sqrt{-q_{i}} T_{i}
$$


where $q_{i}$ is the determinant of the induced metric on the 3-brane. The tension of the 3-brane does not couple to the dilatonic scalar field after dimensional reduction. Thus, we may use the $(6+n)$-dimensional pure Einstein-Maxwell description (2.6) with (2.3) instead of working directly in the 6D Einstein-Maxwell-dilaton system (2.1) with tensional branes (2.8). Given a metric $\mathcal{G}_{M N}$ of the form (2.4) and a gauge field that satisfies (2.5), one automatically obtains $g_{a b}, F_{a b}$, and $\varphi$ that solve the action (2.1). Since the action (2.2) is more simple than (2.1), it is often easier to find a solution of (2.2). Note that in the action (2.6) $n$ is an arbitrary parameter and may no longer be an integer. The right signature for the scalar kinetic term $[n(n+4) \geq 0]$ ensures that $\gamma^{2}$ is nonnegative.

The field equations derived directly from the action (2.1) possess the scaling symmetry

$$
g_{a b} \rightarrow u g_{a b}, \quad \varphi \rightarrow \varphi+\gamma^{-1} \ln u
$$

with constant $u$. Note that this is not a symmetry of the action, as $S^{(6)} \rightarrow u^{2} S^{(6)}$. From the $(6+n)$-dimensional point of view, this symmetry is manifest because we may write

$$
\mathcal{G}_{M N} d \mathcal{X}^{M} d \mathcal{X}^{N}=e^{-n \phi / 2-\ln u} u g_{a b} d x^{a} d x^{b}+\cdots,
$$

and use $u g_{a b}$ and $\phi+(2 / n) \ln u$ instead of $g_{a b}$ and $\phi$.

We would like to obtain six-dimensional axisymmetric warped compactification solutions with conical branes at the points where the geometry pinches off. To this end we make use of the $(6+n)$-dimensional generalization of the exact solution given in Ref. [19], which is obtained by a double Wick rotation from the higher dimensional Reissner-Nordström solution. The metric is written as

$$
\mathcal{G}_{M N} d \mathcal{X}^{M} d \mathcal{X}^{N}=\underbrace{r^{2} \bar{g}_{\alpha \beta} d z^{\alpha} d z^{\beta}}_{(4+n) \mathrm{D}}+\frac{1}{2 \Lambda}\left[\frac{d r^{2}}{\tilde{f}(r)}+\tilde{\beta}^{2} \tilde{f}(r) d \theta^{2}\right],
$$

where

$$
\tilde{f}(r)=\frac{\lambda}{(2+n)(3+n) \Lambda}+\frac{1}{4+n}\left[\frac{C}{r^{3+n}}-\frac{r^{2}}{5+n}-\frac{Q^{2}}{2(3+n) M_{(6+n)}^{4+n} \mathcal{V}_{(n)} \Lambda} \frac{1}{r^{6+2 n}}\right],
$$

and $\bar{g}_{\alpha \beta}$ is any metric that solves the $(4+n)$-dimensional field equations

$$
\bar{R}_{\alpha \beta}[\bar{g}]=\frac{2}{2+n} \lambda \bar{g}_{\alpha \beta} .
$$

The only nonvanishing component of the field strength is given by

$$
\mathcal{F}_{r \theta}=\frac{Q \tilde{\beta}}{2 \Lambda \sqrt{\mathcal{V}_{(n)}}} \frac{1}{r^{4+n}} .
$$

The constant $\tilde{\beta}$ can be absorbed in the angular coordinate $\theta$, but we keep it in the metric.

We assume that $\tilde{f}(r)$ has two positive roots, $r_{+}$and $r_{-}=\alpha r_{+}$with $0<\alpha \leq 1$. In terms of the new coordinate defined by $\chi:=r / r_{+}$, the metric function can be written as $\tilde{f}=r_{+}^{2} f(\chi)$, where

$$
\begin{gathered}
f(\chi)=\frac{1}{(4+n)(5+n)}\left[-\chi^{2}+\frac{1-\alpha^{8+2 n}}{1-\alpha^{3+n}} \frac{1}{\chi^{3+n}}-\frac{\alpha^{3+n}\left(1-\alpha^{5+n}\right)}{1-\alpha^{3+n}} \frac{1}{\chi^{6+2 n}}\right] \\
+\frac{\lambda}{(2+n)(3+n) \Lambda r_{+}^{2}}\left[1-\frac{1}{\chi^{3+n}}\right]\left[1-\frac{\alpha^{3+n}}{\chi^{3+n}}\right] .
\end{gathered}
$$

The parameter $\alpha$ characterizes warping of the bulk. In the case of $\alpha=1$, the bulk is given by a two-dimensional sphere and is often referred to as the rugby-ball compactification.

The rescaling $r_{+}^{2} \bar{g}_{\alpha \beta} d z^{\alpha} d z^{\beta} \rightarrow \bar{g}_{\alpha \beta} d z^{\alpha} d z^{\beta}$ and $\lambda / r_{+}^{2} \rightarrow \lambda$ allows us to write

$$
\mathcal{G}_{M N} d \mathcal{X}^{M} d \mathcal{X}^{N}=\chi^{2} \bar{g}_{\alpha \beta} d z^{\alpha} d z^{\beta}+\frac{1}{2 \Lambda}\left[\frac{d \chi^{2}}{f(\chi)}+\beta^{2} f(\chi) d \theta^{2}\right],
$$

and

$$
\mathcal{F}_{\chi \theta}=\frac{M_{(6+n)}^{2+n / 2} \beta}{\sqrt{2 \Lambda}} \frac{\hat{Q}}{\chi^{4+n}}
$$


where

$$
\hat{Q}:=\left[\frac{3+n}{5+n} \frac{1-\alpha^{5+n}}{1-\alpha^{3+n}}-\frac{4+n}{2+n} \frac{\lambda}{\Lambda}\right]^{1 / 2} \alpha^{(3+n) / 2} .
$$

With this rescaling, $r_{+}$in Eq. (2.15) is absorbed in $\lambda$ and therefore the solution does not have an explicit dependence on $r_{+}$, while $\bar{g}_{\alpha \beta}$ still solves Eq. (2.13).

It is clear from Eq. (2.18) that $\lambda$ is bounded from above:

$$
\lambda \leq \lambda_{\max }(\alpha):=\frac{(2+n)(3+n)}{(4+n)(5+n)} \frac{1-\alpha^{5+n}}{1-\alpha^{3+n}} \Lambda .
$$

A large value of $\lambda$ reduces the strength of flux, and the flux vanishes if the bound is saturated. Such a bound on the brane curvature scale can also be observed in 6D flux compactification models with de Sitter branes [10, 22].

After dimensional reduction $n$ is a parameter of the $6 \mathrm{D}$ theory which is directly related to the dilatonic coupling. In particular, Nishino-Sezgin supergravity $(\gamma=1)$ is reproduced by taking $n \rightarrow \infty$. In this limit the metric function $f(\chi)$ is apparently singular. This is due to a bad choice of the coordinate in the extra direction. A regular expression is obtained by using $\xi:=\chi^{1+n / 2}$ and $\hat{\alpha}:=\alpha^{1+n / 2}$, in terms of which one can write the metric in $(6+n)$ dimensions as

$$
\mathcal{G}_{M N} d \mathcal{X}^{M} d \mathcal{X}^{N}=\xi^{4 /(2+n)} \bar{g}_{\alpha \beta} d z^{\alpha} d z^{\beta}+\frac{\xi^{-n /(2+n)}}{2 \Lambda}\left[\frac{d \xi^{2}}{h(\xi)}+\hat{\beta}^{2} h(\xi) d \theta^{2}\right]
$$

where

$$
\begin{gathered}
h(\xi):=\frac{1}{2(1+\omega)(2+3 \omega)}\left[-\xi^{1+\omega}+\frac{1-\hat{\alpha}^{4(1+\omega)}}{1-\hat{\alpha}^{2+\omega}} \frac{1}{\xi^{1+2 \omega}}-\frac{\hat{\alpha}^{2+\omega}\left(1-\hat{\alpha}^{2+3 \omega}\right)}{1-\hat{\alpha}^{2+\omega}} \frac{1}{\xi^{3(1+\omega)}}\right] \\
+\frac{\lambda}{\Lambda} \frac{\xi^{1-\omega}}{2(2+\omega)}\left[1-\frac{1}{\xi^{2+\omega}}\right]\left[1-\frac{\hat{\alpha}^{2+\omega}}{\xi^{2+\omega}}\right]
\end{gathered}
$$

and $\hat{\beta}:=\omega \beta$ with $\omega:=(1+n / 2)^{-1}$. Clearly, $h$ is regular for $n \rightarrow \infty(\omega=0)$. The field strength is now given by

$$
\mathcal{F}_{\xi \theta}=\frac{M_{(6+n)}^{2+n / 2} \hat{\beta}}{\sqrt{2 \Lambda}} \frac{\hat{Q}}{\xi^{(8+3 n) /(2+n)}} .
$$

We assume that $\theta$ has period $2 \pi$. The constant $\beta$ (or $\hat{\beta}$ ) controls deficit angles at $\chi=1(\xi=1)$ and $\chi=\alpha(\xi=\hat{\alpha})$, which are given, respectively, by

$$
\delta_{1}=2 \pi\left[1-\frac{\beta}{2}\left|f^{\prime}(1)\right|\right]=2 \pi\left[1-\frac{\hat{\beta}}{2}\left|h^{\prime}(1)\right|\right], \quad \delta_{2}=2 \pi\left[1-\frac{\beta}{2}\left|f^{\prime}(\alpha)\right|\right]=2 \pi\left[1-\frac{\hat{\beta}}{2}\left|h^{\prime}(\hat{\alpha})\right|\right] .
$$

Here the prime stands for a derivative with respect to the argument. The conical deficit corresponds to a codimension two brane and the tension is given by $\mathcal{T}_{i} / M_{(6+n)}^{4+n}=\delta_{i}$. Whereas in general there are two branes in the present model, one can choose $\beta$ so that one of the deficit angles vanishes. However, for the warped $\alpha \neq 1$ case it is not possible to make both of the deficit angles zero. Hence, the warped model includes at least one brane. The deficit angles satisfy the constraint

$$
\frac{2 \pi-\delta_{2}}{2 \pi-\delta_{1}}=\left|\frac{h^{\prime}(\hat{\alpha})}{h^{\prime}(1)}\right|\left[=\left|\frac{f^{\prime}(\alpha)}{f^{\prime}(1)}\right|\right]
$$

which therefore places the constraint between the brane tensions.

It is instructive to present here the simplest example: a Minkowski braneworld. The desired solution is generated from the $(4+n)$-dimensional Minkowski metric: $\bar{g}_{\alpha \beta} d z^{\alpha} d z^{\beta}=\eta_{\mu \nu} d x^{\mu} d x^{\nu}+\delta_{m n} d y^{m} d y^{n}$ (and hence $\lambda=0$ ). We identify $e^{\phi}=\chi=\xi^{2 /(2+n)}$ and then the $6 \mathrm{D}$ metric is

$$
g_{a b} d x^{a} d x^{b}=\xi^{2 /\left(1+\gamma^{2}\right)} \eta_{\mu \nu} d x^{\mu} d x^{\nu}+\frac{1}{2 \Lambda}\left[\frac{d \xi^{2}}{h(\xi)}+\hat{\beta}^{2} h(\xi) d \theta^{2}\right] .
$$


The field strength and the scalar field is given, respectively, by

$$
\begin{aligned}
F_{\xi \theta} & =\frac{M_{(6)}^{2} \hat{\beta}}{\sqrt{2 \Lambda}} \frac{\hat{Q}}{\xi^{2\left(2+\gamma^{2}\right) /\left(1+\gamma^{2}\right)}}, \\
\varphi & =\frac{2 \gamma}{1+\gamma^{2}} \ln \xi .
\end{aligned}
$$

Since $\mathcal{T}_{i} / M_{(6+n)}^{4+n}=T_{i} / M_{(6)}^{4}$, the conical deficit and the 3-brane tension are related by $T_{i} / M_{(6)}^{4}=\delta_{i}$. One can check that the above configuration reduces to the known solution in the Einstein-Maxwell model [20, 21] and in Nishino-Sezgin supergravity [23, 24, 25] for the corresponding value of $\gamma$.

\section{EXACT COSMOLOGICAL SOLUTIONS}

Cosmological solutions can be systematically generated from the following Kasner-type metric, ${ }^{2}$

$$
\bar{g}_{\alpha \beta} d z^{\alpha} d z^{\beta}=-d t^{2}+\underbrace{e^{2 A(t)} \delta_{i j} d x^{i} d x^{j}}_{3 \mathrm{D}}+\underbrace{e^{2 B(t)} \delta_{m n} d x^{m} d x^{n}}_{n \mathrm{D}} .
$$

The explicit form of the time-dependent metric functions $A(t)$ and $B(t)$ is derived in detail in Appendix $\mathrm{A}$. Looking at Eq. (2.4) and the metric (2.16) [or (2.20)] with (3.1), we identify $e^{\phi}=\chi e^{B}=\xi^{2 /(2+n)} e^{B}$. Then the 6D metric is found to be

$$
\begin{aligned}
g_{a b} d x^{a} d x^{b} & =\xi^{2 /\left(1+\gamma^{2}\right)}\left[-e^{n B / 2} d t^{2}+e^{2 A+n B / 2} \delta_{i j} d x^{i} d x^{j}\right]+\frac{e^{n B / 2}}{2 \Lambda}\left[\frac{d \xi^{2}}{h(\xi)}+\hat{\beta}^{2} h(\xi) d \theta^{2}\right] \\
& =\xi^{2 /\left(1+\gamma^{2}\right)}\left[-d \tau^{2}+a^{2}(\tau) \delta_{i j} d x^{i} d x^{j}\right]+\frac{b^{2}(\tau)}{2 \Lambda}\left[\frac{d \xi^{2}}{h(\xi)}+\hat{\beta}^{2} h(\xi) d \theta^{2}\right]
\end{aligned}
$$

where we have introduced the proper time on the brane, $\tau=\int e^{n B / 4} d t$, and defined the scale factors of the external and internal spaces as $a(\tau):=e^{A+n B / 4}$ and $b(\tau):=e^{n B / 4}$, respectively. The dilaton is given by

$$
\varphi(\tau, \xi)=\frac{2}{\gamma} \ln b(\tau)+\frac{2 \gamma}{1+\gamma^{2}} \ln \xi
$$

The field strength $F_{\xi \theta}$ is the same as that in the static solution (2.26).

Let us first consider the simple case where the metric functions are given by

$$
A(t)=H_{0} t+A_{0}, \quad B(t)=H_{0} t+B_{0},
$$

with $H_{0}^{2}:=2 \lambda /[(3+n)(2+n)] \neq 0$. In this case it is easy to see that

$$
a(\tau) \propto \tau^{1 / \gamma^{2}}, \quad b(\tau) \propto \tau,
$$

leading to power-law inflation for $\gamma<1$. However, the Nishino-Sezgin supergravity model, $\gamma=1$, does not give an accelerated expansion. The $\gamma=1$ solution was first found by Tolley et al. [27]. While they have not presented an explicit form of the metric of the two-dimensional internal space, Eq. (3.2) shows the full spacetime metric in six dimensions.

In addition to the above trivial solution, we have another type of solutions,

$$
\begin{aligned}
e^{3 A+n B} & =C_{3} \sinh \left[\sqrt{\frac{3+n}{2+n}} \sqrt{2 \lambda}\left(t-t_{0}\right)\right] \\
e^{A-B} & =C_{4}\left\{\tanh \left[\sqrt{\frac{3+n}{2+n}} \sqrt{\frac{\lambda}{2}}\left(t-t_{0}\right)\right]\right\}^{ \pm \sqrt{(2+n) / 3 n}} .
\end{aligned}
$$

\footnotetext{
${ }^{2}$ We derive the main results in this section assuming $n>0$ and hence $\gamma \neq 0$. Obviously, the $n=0$ case does not admit the nontrivial Kasner-type solution and only the de Sitter geometry (as in [19]) is allowed on the brane.
} 
At early times, $t-t_{0} \sim 0$, we have $a \sim \tau^{p_{ \pm}}$and $b \sim \tau^{q_{ \pm}}$where

$$
p_{ \pm}=\frac{3\left(2+\gamma^{2}\right) \pm 2 \sqrt{6} \gamma \sqrt{1+\gamma^{2}}}{3\left(6+5 \gamma^{2}\right)}, \quad q_{ \pm}=\frac{\gamma^{2} \mp \sqrt{6} \gamma \sqrt{1+\gamma^{2}}}{6+5 \gamma^{2}} .
$$

The ranges of $p_{ \pm}$and $q_{ \pm}$are $1 / 3<p_{+} \leq(9+4 \sqrt{3}) / 33 \approx 0.48,1 / 3>p_{-} \geq(9-4 \sqrt{3}) / 33 \approx 0.063,0>q_{+} \geq$ $(1-2 \sqrt{3}) / 11 \approx-0.22$, and $0<q_{-} \leq(1+2 \sqrt{3}) / 11 \approx 0.41$. Note that $q_{+}$is negative and hence in this case the compact two-dimensional space is initially contracting. At late times, $t \rightarrow \infty$, we have $a \sim \tau^{1 / \gamma^{2}}$ and $b \sim \tau$. Therefore, the solution (3.5) is the late-time attractor. This attractor behavior on the $4 \mathrm{D}$ brane emerges as a consequence of Wald's cosmic no hair theorem [38] for the $(4+n)$-dimensional metric $\bar{g}_{\alpha \beta}$ : the initially expanding Bianchi-type metric (3.1) isotropizes in the presence of the positive cosmological constant $\lambda$.

The cosmological dynamics can also be understood in terms of the $4 \mathrm{D}$ effective theory in the zero-mode sector. In Appendix B we show that the induced metric on the brane can be derived from the Einstein-scalar field action with an exponential potential up to conformal transformations. Given generic initial conditions for the "position" and "velocity" of the scalar field, the scale factor and the time-dependence of $\varphi$ are obtained from (3.6) and (3.7). In this case the two of the three integration constants correspond to the initial position and velocity of the scalar field, whereas the third integration constant corresponds to the overall normalization of the scale factor. The solution (3.5) is realized for the special choice of the initial velocity (depending on the initial position), as (3.4) has only two integration constants. The power-law solution obtained from (3.4) is the late-time attractor for the exponential potential of the scalar field. The cosmic expansion looks very different in a different frame, but the discussion here in terms of the 4D effective theory (B3) is still helpful.

The dynamics of the $\lambda=0$ model is the same as the early-time behavior of the general $\lambda \neq 0$ case: $a \sim \tau^{p_{ \pm}}$and $b \sim \tau^{q_{ \pm}}$. From Eq. (3.8) we see that the $\gamma=1$ case gives the scale factor identical to that in [36, 37]. One should note here that the geometry on the brane is independent of the warping parameter $\alpha$ and the brane tension. They only affect the bulk geometry. For this reason, it is not surprising that we can reproduce the scale factor of the model without warping and branes [36, 37] just by taking $\gamma=1$.

\section{COSMOLOGICAL TENSOR PERTURBATIONS}

We will be discussing the behavior of perturbations in the cosmological background solution constructed in the previous section. Here we make use of the $(6+n)$-dimensional description again, and focus on the axisymmetric tensor perturbations. ${ }^{3}$ The perturbed metric is given by

$$
\left(\mathcal{G}_{M N}^{(0)}+\delta \mathcal{G}_{M N}^{(1)}\right) d \mathcal{X}^{M} d \mathcal{X}^{N}=\chi^{2}\left[-d t^{2}+e^{2 A}\left(\delta_{i j}+h_{i j}\right) d x^{i} d x^{j}+e^{2 B} \delta_{m n} d y^{m} d y^{n}\right]+\frac{1}{2 \Lambda}\left[\frac{d \chi^{2}}{f}+\beta^{2} f d \theta^{2}\right],
$$

where $h_{i j}=h_{i j}(t, \mathbf{x}, \chi)$ and $h_{i}{ }^{i}=\partial^{j} h_{i j}=0$. The $(6+n)$-dimensional Einstein equations give

$$
e^{-3 A-n B} \partial_{t}\left(e^{3 A+n B} \partial_{t} h_{i j}\right)-e^{-2 A} \nabla^{2} h_{i j}=2 \Lambda \chi^{-2-n} \partial_{\chi}\left(\chi^{4+n} f \partial_{\chi} h_{i j}\right),
$$

which is separable. In the Fourier space, the general solution can be written as

$$
h_{i j}=\sum_{m} \psi_{m}(t) \Omega_{m}(\chi) e_{i j}^{\mathrm{TT}}(\mathbf{k} ; \mathbf{x})
$$

where $e_{i j}^{\mathrm{TT}}$ is the transverse traceless tensor harmonics which satisfies $\nabla^{2} e_{i j}^{\mathrm{TT}}=-k^{2} e_{i j}^{\mathrm{TT}}$.

The mode solutions $\psi_{m}$ and $\Omega_{m}$ obey

$$
\begin{aligned}
{\left[\frac{d^{2}}{d t^{2}}+(3 \dot{A}+n \dot{B}) \frac{d}{d t}+\frac{k^{2}}{e^{2 A}}+\mu_{m}^{2}\right] \psi_{m} } & =0 \\
\frac{1}{\chi^{2+n}} \frac{d}{d \chi}\left(\chi^{4+n} f \frac{d}{d \chi} \Omega_{m}\right)+\frac{\mu_{m}^{2}}{2 \Lambda} \Omega_{m} & =0
\end{aligned}
$$

\footnotetext{
3 Perturbations are decomposed into scalar, vector, and tensor parts according to their transformation properties with respect to the three-dimensional space. Scalar and vector perturbations will be investigated in a forthcoming paper.
} 
where the dot denotes a derivative with respect to $t$ and $\mu_{m}^{2}$ is a separation eigenvalue. At the poles the metric function $f$ vanishes. Therefore, the boundary conditions for $\Omega_{m}$ are

$$
\chi^{2} f^{\prime} \Omega_{m}^{\prime}+\frac{\mu_{m}^{2}}{2 \Lambda} \Omega_{m}=0 \quad \text { at } \quad \chi=1, \alpha .
$$

Two things should be remarked here. First, there always exist a zero mode $\left(\mu_{0}^{2}=0\right)$ :

$$
\Omega_{0}=\text { const. }
$$

Second, there are no tachyonic modes $\left(\mu_{m}^{2}<0\right)$ in the spectrum. This can be shown as follows. Using Eq. (4.5) we obtain

$$
\frac{\mu_{m}^{2}}{2 \Lambda} \int_{\alpha}^{1} \chi^{2+n} \Omega_{m}^{2} d \chi=-\int_{\alpha}^{1} \Omega_{m}\left(\chi^{4+n} f \Omega_{m}^{\prime}\right)^{\prime} d \chi=\int_{\alpha}^{1} \chi^{4+n} f\left(\Omega_{m}^{\prime}\right)^{2} d \chi \geq 0
$$

where we used $f(1)=f(\alpha)=0$ and assumed the regularity of $\Omega_{m}$ and $\Omega_{m}^{\prime}$ at the boundaries. Thus, $\mu_{m}^{2}$ is not negative. The equality holds only for $\Omega_{m}^{\prime}=0$, which is definitely the zero mode.

Eq. (4.4) can be written in terms of the conformal time coordinate $\eta:=\int e^{-A} d t$ as

$$
\left[\frac{d^{2}}{d \eta^{2}}+\left(2 \mathcal{H}+\frac{n}{2} \frac{d B}{d \eta}\right) \frac{d}{d \eta}+k^{2}+e^{-n B / 2} \mu_{m}^{2} a^{2}\right] \psi_{m}=0
$$

where $\mathcal{H}:=a^{-1} d a / d \eta$ is the conformal Hubble parameter on the brane. We see from Eq. (4.9) that the Kaluza-Klein (KK) mass with respect to observers on the $4 \mathrm{D}$ brane is time-dependent and is given by

$$
M_{m}^{2}(\eta):=e^{-n B(\eta) / 2} \mu_{m}^{2}
$$

As for the zero mode, Eq. (4.9) gives the standard $4 \mathrm{D}$ equation for a massless graviton except for the extra term $d B / d \eta$. The presence of this term reflects the fact that the effective theory for the zero-mode sector is given by a scalar-tensor gravity, as is argued in Appendix B.

We are mainly interested in the inflationary attractor solution (3.4). In this case, Eq. (4.9) reduces to

$$
\left(\frac{d^{2}}{d \eta^{2}}-\frac{2+n}{\eta} \frac{d}{d \eta}+k^{2}+\frac{\mu_{m}^{2}}{H_{0}^{2} \eta^{2}}\right) \psi_{m}=0
$$

which can be solved exactly for any value of $\mu_{m}^{2}$. The general solution is given by

$$
\psi_{m}=(-k \eta)^{(3+n) / 2} Z_{i \nu_{m}}(-k \eta),
$$

where $Z_{\nu}$ is a linear combination of Bessel functions of order $\nu$ and

$$
\nu_{m}^{2}:=\frac{\mu_{m}^{2}}{H_{0}^{2}}-\frac{(3+n)^{2}}{4} .
$$

The late-time asymptotic behavior of (4.12) depends on the value of $\mu_{m}^{2}$. "Heavy" modes with $\mu_{m}^{2} \geq \mu_{c}^{2}$, where

$$
\mu_{c}^{2}:=\frac{(3+n) \lambda}{2(2+n)}
$$

decay rapidly as $\left|\psi_{m}\right| \sim a^{-2(3+n) /(4+n)}$ at late times $(-k \eta \rightarrow 0)$. "Light" modes with $\mu_{m}^{2}<\mu_{c}^{2}$ decay more slowly,

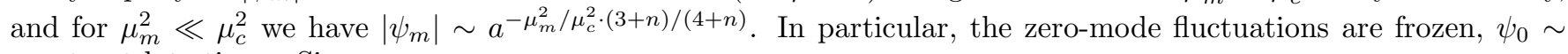
const., at late times. Since

$$
\frac{M_{m}^{2} a^{2}}{\mathcal{H}^{2}}=\left[\frac{2(3+n)}{(4+n)}\right]^{2} \frac{\mu_{m}^{2}}{\mu_{c}^{2}},
$$

heavy (light) modes in the $(6+n)$-dimensional description are also heavy (light) for a $4 \mathrm{D}$ brane observer. 

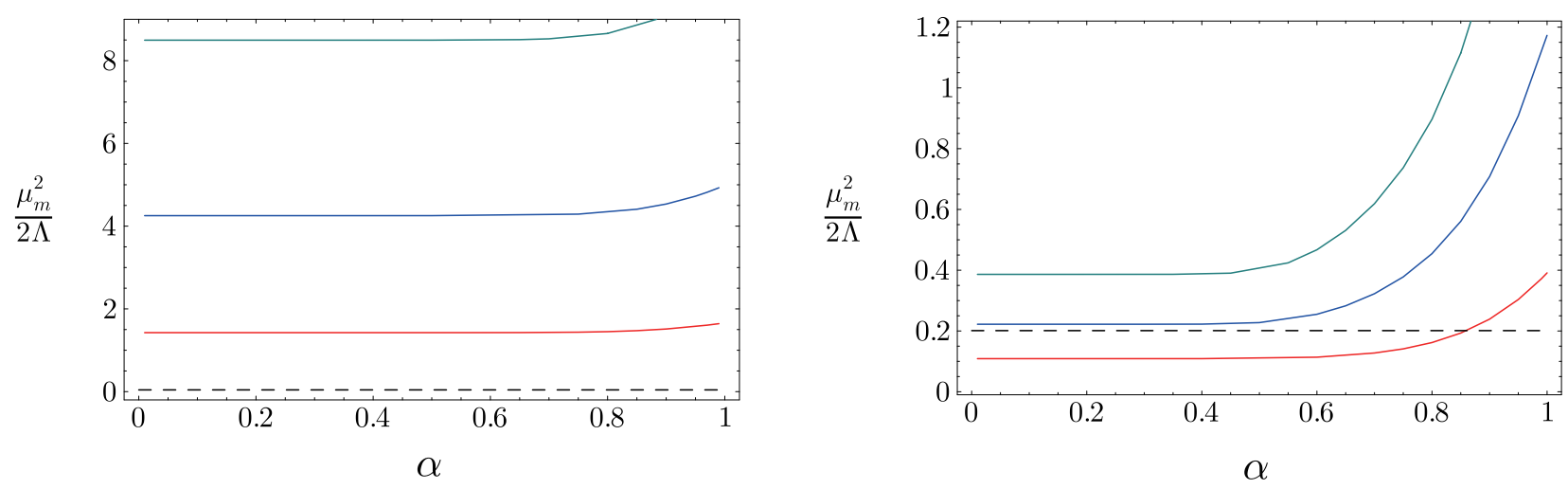

FIG. 1: Eigenvalues of the first three Kaluza-Klein modes, $\mu_{1}^{2}, \mu_{2}^{2}$, and $\mu_{3}^{2}$, versus $\alpha$ for $n=10$. The parameter is given by $\lambda / \Lambda=0.156$ (left) and $\lambda / \Lambda=0.74256$ (right). The dashed line indicates $\mu_{c}^{2}$.

Now we are to determine the mass spectrum of KK modes $\left\{\mu_{1}^{2}, \mu_{2}^{2}, \cdots\right\}$ numerically. The spectrum depends on $n$ and the parameters of the background solution, $\alpha$ and $\lambda$. Given a set of parameters, we numerically solve Eq. (4.5) supplemented with the condition $\Omega(1)=1$ and $\Omega^{\prime}(1)=-\mu^{2} /\left[2 \Lambda f^{\prime}(1)\right]$. Then we have

$$
I\left(\mu^{2}\right):=\left[\alpha^{2} f^{\prime} \Omega^{\prime}+\frac{\mu^{2}}{2 \Lambda} \Omega\right]_{\chi=\alpha}
$$

as a function of $\mu^{2}$. The mass spectrum is given by zeros of $I\left(\mu^{2}\right)$.

Examples of $\mu_{m}^{2}$ are shown in Fig. 1. As is clear, $\mu_{m}^{2}$ is an increasing function of $\alpha$. One finds that $\mu_{1}^{2}$ can be smaller than $\mu_{c}^{2}$ for a relatively large value of $\lambda$. To see this more closely, we plot in Fig. 2 the lightest KK mass $\mu_{1}^{2}$ in the limit of $\alpha \rightarrow 0$ as a function of $\lambda$. We find that for $n \sim 1$ we have $\mu_{1}^{2} \sim \mu_{c}^{2}$ when $\lambda$ is close to its maximum value, while larger $n$ tends to give a very light mode for large $\lambda$.

If we have very light modes in the spectrum, such modes can be excited during the period of inflation and decay slowly. This is certainly an interesting situation, but is not favored for the following reason. According to Ref. [39], a large expansion rate in the external coordinates generally has the effect of destablizing de Sitter compactifications. In fact, the presence of $\lambda$ (corresponding to the $(4+n)$-dimensional Hubble rate) decreases the strength of flux as seen in Eq. (2.18). It was also confirmed directly in 22] that in the $6 \mathrm{D}$ flux compactification model there appears a tachyonic mode in the scalar sector of perturbations when the expansion on the brane is too large (but still less than the upper bound given by Eq. (2.19) with $n=0) .{ }^{4}$ From this we may expect that the $(6+n)$-dimensional Einstein-Maxwell model will also be unstable for large $\lambda$, leading to the instability of the 6D Einstein-Maxwell-dilaton model since the two descriptions are equivalent. In light of this, it is natural to consider that smaller $\lambda$ is favored also in the present model. For sufficiently small $\lambda$ all the KK modes are heavy enough to decay rapidly at late times.

For understanding the behavior of KK perturbations in more detail, it would be helpful to provide an analytic argument on the mode functions and eigenvalues in the special case of $\alpha=1$. Rewriting Eq. (4.5) in terms of the new coordinate $w:=(2 \chi-1-\alpha) /(1-\alpha)$ and then taking the limit $\alpha \rightarrow 1$, we get the equation

$$
\left(1-\frac{3+n}{4+n} \Delta\right) \frac{d}{d w}\left[\left(1-w^{2}\right) \frac{d}{d w} \Omega_{m}\right]+\frac{\mu_{m}^{2}}{2 \Lambda} \Omega_{m}=0
$$

subject to the boundary conditions

$$
\mp 2\left(1-\frac{3+n}{4+n} \Delta\right) \frac{d \Omega_{m}}{d w}+\frac{\mu_{m}^{2}}{2 \Lambda} \Omega_{m}=0 \quad \text { at } \quad w= \pm 1,
$$

where $\Delta:=\lambda / \lambda_{\max }(1)$. The mode solution is given up to the overall normalization by

$$
\Omega_{m}=P_{m}(w), \quad \frac{\mu_{m}^{2}}{2 \Lambda}=m(m+1)\left(1-\frac{3+n}{4+n} \Delta\right)
$$

\footnotetext{
${ }^{4}$ We should remark that in [22] scalar, vector, and tensor perturbations are defined with respect to the 4D de Sitter spacetime
} 

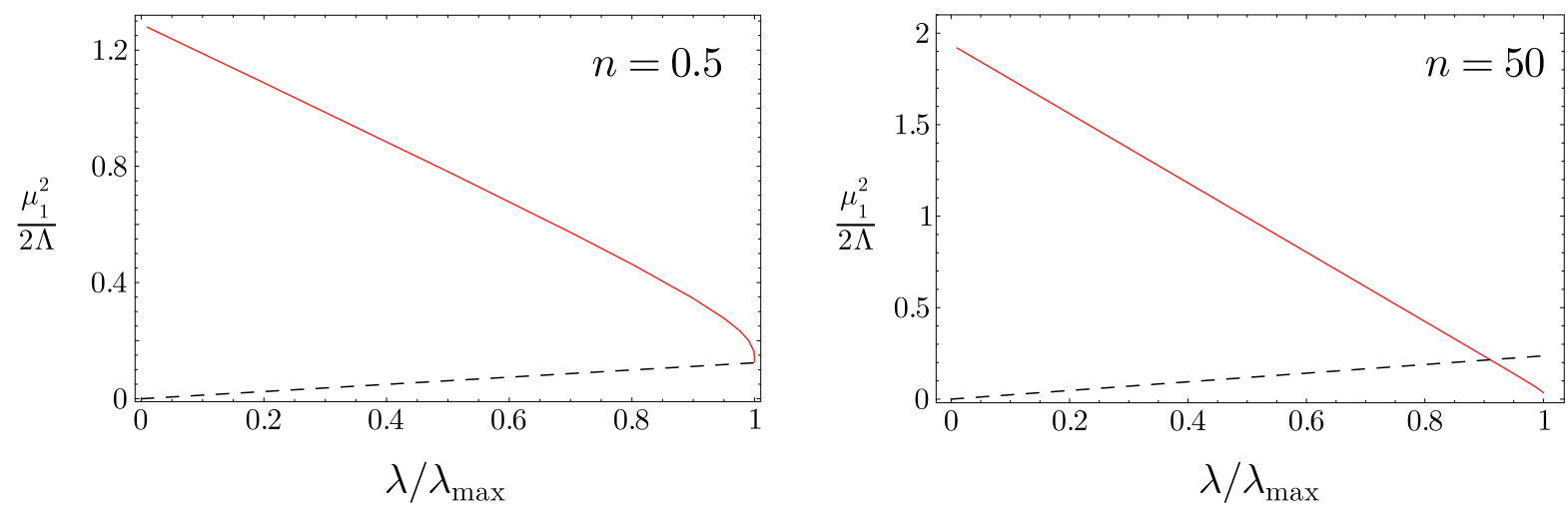

FIG. 2: First Kaluza-Klien eigenvalue in the limit of $\alpha \rightarrow 0$ versus $\lambda / \lambda_{\max }(0)$. The dashed line indicates $\mu_{c}^{2}$.

where $P_{m}$ is the Legendre function of the first kind of order $m$ and $m=0,1,2, \cdots$. We checked that this analytic result agrees with our numerical calculations in the $\alpha \rightarrow 1$ limit.

It follows from the mass spectrum (4.19) that

$$
\frac{\mu_{m}^{2}}{\mu_{c}^{2}}=4 m(m+1)\left(\frac{4+n}{3+n} \frac{1}{\Delta}-1\right) .
$$

Very light modes emerge if and only if $\lambda \approx \lambda_{\max }(1)$ and $n$ is very large.

Finally, we shall make a brief comment on scalar perturbations in the present model. Our $(6+n)$-dimensional description would be especially powerful when studying scalar perturbations, as is the case in the Randall-Sundrumtype braneworld inflation model with a bulk scalar field [29]. This is mainly because the extra scalar degree of freedom in six dimensions is encoded in a metric perturbation in $(6+n)$-dimensions. This fact allows one to find a convenient gauge in which both perturbation equations and boundary conditions become simple enough to handle. The emergence of a tachyonic perturbation mode in the scalar sector in the 6D Einstein-Maxwell model [22] implies that such instability may arise in general $(6+n)$-dimensions, and hence the scalar perturbations need a detailed investigation (see also Refs. [40, 41] for scalar perturbations in the Minkowski brane models in the context of NishinoSezgin supergravity). This issue will be considered carefully in a forthcoming publication.

\section{SUMMARY}

In this paper we have proposed a new systematic method quite useful for studying brane cosmology in warped flux compactifications. The crucial key is that the Einstein-Maxwell-dilaton system in six dimensions is equivalent to the $(6+n)$-dimensional pure Einstein-Maxwell system under our metric ansatz. Using this fact, we have demonstrated that time-dependent warped compactification solutions can be obtained easily. Our "seed" is essentially the double Wick rotated black hole solution in Einstein-Maxwell theory, which we believe is more simple and familiar. For a general dilatonic coupling $\gamma$, we have found a power-law inflationary solution and two nontrivial time-dependent solutions. The former solution was turned out to be the late-time attractor for $\lambda \neq 0$. In the case of Nishino-Sezgin supergravity $(\gamma=1)$, the power-law solution reduces to the noninflating solution of [27]. Here we have obtained the explicit form of the metric of the internal two-dimensional space. For the particular choice of the parameter, $\lambda=0$, we have seen that our solution reproduces the cosmological solution of [36, 37] (but with warped bulk geometry and conical deficits, which do not affect the geometry of the external space).

We have also investigated the dynamics of tensor perturbations using our $(6+n)$-dimensional description. We obtained the separable equation of motion for axisymmetric tensor perturbations, and showed that there always exists a zero mode, whereas we have no tachyonic modes in the spectrum. For relatively small $\lambda$, which is likely from the stability consideration, KK modes are too heavy to be excited during inflation.

\section{Acknowledgments}

We wish to thank Osamu Seto for comments. TK is supported by the JSPS under Contract No. 19-4199. The work of MM was supported by the project "Transregio (Dark Universe)" at the ASC. 


\section{APPENDIX A: DERIVATION OF THE KASNER-TYPE METRIC}

In this appendix we present a detailed derivation of the Kanser-type metric used in the main text. The metric takes the form

$$
\bar{g}_{\alpha \beta} d z^{\alpha} d z^{\beta}=-d t^{2}+\underbrace{e^{2 A(t)} \delta_{i j} d x^{i} d x^{j}}_{3 \mathrm{D}}+\underbrace{e^{2 B(t)} \delta_{m n} d x^{m} d x^{n}}_{n \mathrm{D}} .
$$

The field equations $\bar{R}_{\alpha \beta}=[2 /(2+n)] \lambda \bar{g}_{\alpha \beta}$ read

$$
\begin{aligned}
(t t): & 3\left(\ddot{A}+\dot{A}^{2}\right)+n\left(\ddot{B}+\dot{B}^{2}\right) & =\frac{2 \lambda}{2+n}, \\
(i j): & \ddot{A}+\dot{A}(3 \dot{A}+n \dot{B}) & =\frac{2 \lambda}{2+n}, \\
(m n): & \ddot{B}+\dot{B}(3 \dot{A}+n \dot{B}) & =\frac{2 \lambda}{2+n},
\end{aligned}
$$

where the dot denotes a derivative with respect to $t$. From Eqs. (A3) and (A4) we get $\left(e^{3 A+n B}\right)^{\cdot *}=\tilde{\lambda} e^{3 A+n B}$ and $\left[(\dot{A}-\dot{B}) e^{3 A+n B}\right]^{\cdot}=0$, where $\tilde{\lambda}:=[2(3+n) /(2+n)] \lambda$. For $\lambda \neq 0$, integration of these two equations gives

$$
\begin{aligned}
& e^{3 A+n B}=C_{1} e^{\sqrt{\tilde{\lambda}} t}+C_{2} e^{-\sqrt{\tilde{\lambda}} t}, \\
& (\dot{A}-\dot{B}) e^{3 A+n B}=C_{3} \sqrt{\frac{(2+n) \tilde{\lambda}}{3 n}} .
\end{aligned}
$$

Substituting this result into Eq. (A2), we obtain

$$
4 C_{1} C_{2}+C_{3}^{2}=0
$$

If $C_{3}=0$, the relation (A7) implies that $C_{2}=0$ (We are interested in an expanding case). Then we have the de Sitter solution:

$$
A(t)=H_{0} t+A_{0}, \quad B(t)=H_{0} t+B_{0},
$$

with $H_{0}^{2}=2 \lambda /[(3+n)(2+n)]$. This solution has two integration constants, $A_{0}$ and $B_{0}$. If $C_{3} \neq 0$, we may write $C_{1}= \pm\left(C_{3} / 2\right) e^{-\sqrt{\tilde{\lambda}} t_{0}}$ and $C_{2}=\mp\left(C_{3} / 2\right) e^{\sqrt{\tilde{\lambda}} t_{0}}$, where $t_{0}$ is a constant. In this case we have

$$
\begin{aligned}
e^{3 A+n B} & =C_{3} \sinh \left[\sqrt{\tilde{\lambda}}\left(t-t_{0}\right)\right], \\
e^{A-B} & =C_{4}\left\{\tanh \left[\frac{\sqrt{\tilde{\lambda}}}{2}\left(t-t_{0}\right)\right]\right\}^{ \pm \sqrt{(2+n) / 3 n}},
\end{aligned}
$$

where we have introduced another integration constant $C_{4}$ in addition to $C_{3}$ and $t_{0}$. This solution has three integration constants, in contrast to the de Sitter case.

In the case of $\lambda=0$ it is easy to find

$$
\begin{aligned}
e^{3 A+n B} & =C_{1}\left(t-t_{0}\right), \\
e^{A-B} & =C_{2}\left(t-t_{0}\right)^{ \pm \sqrt{(2+n) / 3 n}} .
\end{aligned}
$$

The behavior of the solution is the same as that of (A9) and (A10) at $t \sim t_{0}$.

\section{APPENDIX B: EFFECTIVE THEORY IN THE ZERO MODE SECTOR}

The effective four-dimensional action governing the induced geometry on the brane at $\xi=1$ is

$$
S_{\mathrm{eff}}=\frac{M_{(4)}^{2}}{2} \int d^{4} x \sqrt{-q}\left[e^{n B / 2} R^{(4)}-\omega_{\mathrm{BD}} \frac{n^{2}}{4} e^{n B / 2} q^{\mu \nu} \partial_{\mu} B \partial_{\nu} B-2 \lambda\right],
$$


with $\omega_{\mathrm{BD}}:=\frac{8+n}{2 n}$. This can be obtained from the Einstein-Maxwell action (2.2) simply by substituting the ansatz

$$
\mathcal{G}_{M N} d \mathcal{X}^{M} \mathcal{X}^{N}=\chi^{2}[\underbrace{e^{-n B(x) / 2} q_{\mu \nu}(x) d x^{\mu} d x^{\nu}}_{4 \mathrm{D}}+\underbrace{e^{2 B(x)} \delta_{m n} d y^{m} d y^{n}}_{n \mathrm{D}}]+\frac{1}{2 \Lambda}\left(\frac{d \chi^{2}}{f}+\beta^{2} f d \theta^{2}\right),
$$

and $\mathcal{F}_{\chi \theta}=M_{(6+n)}^{2+n / 2} \beta / \sqrt{2 \Lambda} \cdot \hat{Q} / \chi^{4+n}\left[\right.$ Eq. (2.17)] , with the definition of the four-dimensional Planck mass $M_{(4)}^{2}:=$ $M_{(6)}^{4} \int \sqrt{\mathcal{G}_{\chi \chi} \mathcal{G}_{\theta \theta}} d \chi d \theta$.

The conformal transformation $\tilde{q}_{\mu \nu}=e^{n B / 2} q_{\mu \nu}$ leads to

$$
S_{\mathrm{eff}}=\frac{M_{(4)}^{2}}{2} \int d^{4} x \sqrt{-\tilde{q}}\left[\tilde{R}^{(4)}-\frac{n(2+n)}{2} \tilde{q}^{\mu \nu} \partial_{\mu} B \partial_{\nu} B-2 \lambda e^{-n B}\right] .
$$

Thus the zero-mode sector of our model can be described by the Einstein-scalar field system with an exponential potential.

[1] See, e.g., V. A. Rubakov, Phys. Usp. 44, 871 (2001) [Usp. Fiz. Nauk 171, 913 (2001)] arXiv:hep-ph/0104152].

[2] N. Arkani-Hamed, S. Dimopoulos and G. R. Dvali, Phys. Lett. B 429, 263 (1998) arXiv:hep-ph/9803315; I. Antoniadis, N. Arkani-Hamed, S. Dimopoulos and G. R. Dvali, Phys. Lett. B 436, 257 (1998) arXiv:hep-ph/9804398; I. Antoniadis and C. Bachas, Phys. Lett. B 450, 83 (1999) arXiv:hep-th/9812093.

[3] J. W. Chen, M. A. Luty and E. Ponton, JHEP 0009, 012 (2000) arXiv:hep-th/0003067; S. M. Carroll and M. M. Guica, arXiv:hep-th/0302067; I. Navarro, JCAP 0309, 004 (2003) arXiv:hep-th/0302129; Y. Aghababaie, C. P. Burgess, S. L. Parameswaran and F. Quevedo, Nucl. Phys. B 680, 389 (2004) arXiv:hep-th/0304256].

[4] I. Navarro, Class. Quant. Grav. 20, 3603 (2003) arXiv:hep-th/0305014]; H. P. Nilles, A. Papazoglou and G. Tasinato, Nucl. Phys. B 677, 405 (2004) arXiv:hep-th/0309042; H. M. Lee, Phys. Lett. B 587, 117 (2004) arXiv:hep-th/0309050; J. Vinet and J. M. Cline, Phys. Rev. D 70, 083514 (2004) arXiv:hep-th/0406141; J. Garriga and M. Porrati, JHEP 0408, 028 (2004) arXiv:hep-th/0406158.

[5] L. Randall and R. Sundrum, Phys. Rev. Lett. 83, 3370 (1999) arXiv:hep-ph/9905221]; L. Randall and R. Sundrum, Phys. Rev. Lett. 83, 4690 (1999) arXiv:hep-th/9906064.

[6] P. Binetruy, C. Deffayet and D. Langlois, Nucl. Phys. B 565, 269 (2000) arXiv:hep-th/9905012 ; P. Binetruy, C. Deffayet, U. Ellwanger and D. Langlois, Phys. Lett. B 477, 285 (2000) arXiv:hep-th/9910219; S. Mukohyama, Phys. Lett. B 473, 241 (2000) arXiv:hep-th/9911165; D. Ida, JHEP 0009, 014 (2000) arXiv:gr-qc/9912002; P. Kraus, JHEP 9912, 011 (1999) arXiv:hep-th/9910149.

[7] P. Kanti, R. Madden and K. A. Olive, Phys. Rev. D 64, 044021 (2001) arXiv:hep-th/0104177]; J. M. Cline, J. Descheneau, M. Giovannini and J. Vinet, JHEP 0306, 048 (2003) arXiv:hep-th/0304147; P. Bostock, R. Gregory, I. Navarro and J. Santiago, Phys. Rev. Lett. 92, 221601 (2004) arXiv:hep-th/0311074; Z. C. Wu, Phys. Lett. B 612, 115 (2005) arXiv:hep-th/0405249.

[8] E. Papantonopoulos, arXiv:gr-qc/0601011.

[9] M. Peloso, L. Sorbo and G. Tasinato, Phys. Rev. D 73, 104025 (2006) arXiv:hep-th/0603026.

[10] B. Himmetoglu and M. Peloso, arXiv:hep-th/0612140.

[11] E. Papantonopoulos, A. Papazoglou and V. Zamarias, JHEP 0703, 002 (2007) arXiv:hep-th/0611311.

[12] T. Kobayashi and M. Minamitsuji, Phys. Rev. D 75, 104013 (2007) arXiv:hep-th/0703029.

[13] N. Kaloper and D. Kiley, arXiv:hep-th/0703190.

[14] D. Yamauchi and M. Sasaki, arXiv:0705.2443 [gr-qc].

[15] C. P. Burgess, D. Hoover and G. Tasinato, arXiv:0705.3212 [hep-th].

[16] C. Charmousis and R. Zegers, JHEP 0508, 075 (2005) arXiv:hep-th/0502170; C. Charmousis and R. Zegers, Phys. Rev. D 72, 064005 (2005) arXiv:hep-th/0502171.

[17] H. Nishino and E. Sezgin, Phys. Lett. B 144, 187 (1984); H. Nishino and E. Sezgin, Nucl. Phys. B 278, 353 (1986).

[18] A. Salam and E. Sezgin, Phys. Lett. B 147, 47 (1984).

[19] S. Mukohyama, Y. Sendouda, H. Yoshiguchi and S. Kinoshita, JCAP 0507, 013 (2005) arXiv:hep-th/0506050.

[20] H. Yoshiguchi, S. Mukohyama, Y. Sendouda and S. Kinoshita, JCAP 0603, 018 (2006) arXiv:hep-th/0512212.

[21] Y. Sendouda, S. Kinoshita and S. Mukohyama, Class. Quant. Grav. 23, 7199 (2006) arXiv:hep-th/0607189.

[22] S. Kinoshita, Y. Sendouda and S. Mukohyama, arXiv:hep-th/0703271

[23] Y. Aghababaie et al., JHEP 0309, 037 (2003) arXiv:hep-th/0308064.

[24] G. W. Gibbons, R. Guven and C. N. Pope, Phys. Lett. B 595, 498 (2004) arXiv:hep-th/0307238.

[25] C. P. Burgess, F. Quevedo, G. Tasinato and I. Zavala, JHEP 0411, 069 (2004) arXiv:hep-th/0408109.

[26] A. J. Tolley, C. P. Burgess, D. Hoover and Y. Aghababaie, JHEP 0603, 091 (2006) arXiv:hep-th/0512218.

[27] A. J. Tolley, C. P. Burgess, C. de Rham and D. Hoover, New J. Phys. 8, 324 (2006) arXiv:hep-th/0608083. 
[28] L. Anchordoqui, H. Goldberg, S. Nawata and C. Nunez, arXiv:0704.0928 [hep-ph].

[29] T. Kobayashi and T. Tanaka, Phys. Rev. D 69, 064037 (2004) arXiv:hep-th/0311197.

[30] A. Lukas, B. A. Ovrut, K. S. Stelle and D. Waldram, Phys. Rev. D 59, 086001 (1999) arXiv:hep-th/9803235; A. Lukas, B. A. Ovrut and D. Waldram, Phys. Rev. D 60, 086001 (1999) arXiv:hep-th/9806022.

[31] H. S. Reall, Phys. Rev. D 59, 103506 (1999) arXiv:hep-th/9809195.

[32] S. Kachru, M. B. Schulz and E. Silverstein, Phys. Rev. D 62, 045021 (2000) arXiv:hep-th/0001206; M. Cvetic, H. Lu and C. N. Pope, Phys. Rev. D 63, 086004 (2001) arXiv:hep-th/0007209; V. Bozza, M. Gasperini and G. Veneziano, arXiv:hep-th/0106019.

[33] H. Ochiai and K. Sato, Phys. Lett. B 503, 404 (2001) arXiv:hep-th/0010163; A. Feinstein, K. E. Kunze and M. A. VazquezMozo, Phys. Rev. D 64, 084015 (2001) arXiv:hep-th/0105182; C. Charmousis, Class. Quant. Grav. 19, 83 (2002) arXiv:hep-th/0107126.

[34] K. Koyama and K. Takahashi, Phys. Rev. D 67, 103503 (2003) arXiv:hep-th/0301165]; K. Koyama and K. Takahashi, Phys. Rev. D 68, 103512 (2003) arXiv:hep-th/0307073; H. Yoshiguchi and K. Koyama, Phys. Rev. D 70, 043513 (2004) arXiv:hep-th/0403097.

[35] S. Mukohyama and A. Coley, Phys. Rev. D 69, 064029 (2004) arXiv:hep-th/0310140.

[36] K. i. Maeda and H. Nishino, Phys. Lett. B 154, 358 (1985).

[37] K. i. Maeda and H. Nishino, Phys. Lett. B 158, 381 (1985).

[38] R. W. Wald, Phys. Rev. D 28, 2118 (1983).

[39] C. R. Contaldi, L. Kofman and M. Peloso, JCAP 0408, 007 (2004) arXiv:hep-th/0403270.

[40] H. M. Lee and A. Papazoglou, Nucl. Phys. B 747, 294 (2006) [Erratum-ibid. B 765, 200 (2007)] arXiv:hep-th/0602208].

[41] C. P. Burgess, C. de Rham, D. Hoover, D. Mason and A. J. Tolley, JCAP 0702, 009 (2007) arXiv:hep-th/0610078. 